Maternidad subrogada, autonomía y tecnología. Pensando la economía de las propiedades vitales

Candela Rocío Heredia

http://perio.unlp.edu.ar/ojs/index.php/question/article/view/4652

Cita sugerida: Heredia, C. (2018). Maternidad subrogada, autonomía y tecnología. Pensando la economía de las propiedades vitales. Question, 1(59), e082. doi:https://doi.org/10.24215/16696581e082

\title{
Maternidad subrogada, autonomía y tecnología. Pensando la economía de las propiedades vitales
}

\author{
Subrogated motherhood, autonomy and technology. Thinking the \\ economy of vital properties
}

Candela Rocío Heredia candelarheredia@gmail.com

http://orcid.org/0000-0002-4571-984X

Instituto de Investigaciones Gino Germani; Facultad de

Ciencias Sociales; Universidad de Buenos Aires/Consejo Nacional de Investigaciones Científicas y Técnicas (Argentina)

\section{Resumen}

El presente trabajo trata sobre los discursos de autonomía en la maternidad subrogada. Se analiza un sitio web donde se publicita y gestiona la maternidad subrogada teniendo en cuenta los apartes del sociólogo Nikolas Rose para pensar la economía de las propiedades vitales. Este artículo se aborda desde una perspectiva decolonial del poder, con el fin de analizar en clave histórica el lugar de los discursos en la nueva geopolítica.

Palabras clave: discursos; autonomía; maternidad subrogada; tecnomedicina; bioeconomía. 
Abstract

The work is about the discourses of autonomy in surrogate parenthood. A website where surrogate parenthood is publicized and managed is analyzed, taking into account the sociologist Nikolas Rose's asides to think about the economics of vital properties. This article has a decolonial perspective of power to see in historical terms the place of discourses in the new geopolitics.

Keywords: discourses of autonomy; surrogate parenthood; tecnomedicine; bioeconomy.

Las formas de maternar/paternar han variado según los contextos históricos y las sociedades. En los últimos años en las sociedades occidentales modernas, con las nuevas tecnologías, las formas de maternar/paternar han sufrido profundas transformaciones. A partir de los aportes de Nikolas Rose (2012), este ensayo busca indagar en torno a las relaciones entre el discurso de autonomía y las prácticas de maternidad subrogada y el surgimiento de las nuevas tecnologías que la hacen posible. En el nuevo campo geopolítico donde la biopolítica y la bioeconomía se entrelazan y toman rumbos sorpresivos, se hace propicio pensar en las maneras en que se expresan dichas relaciones y la producción de discursos que las legitiman. Desde este enfoque, indagaré en concreto una empresa de medicina reproductiva llamada Procrearte para poder asir esa economía de las propiedades vitales de los procesos vivos y las proposiciones discursivas.

La perspectiva decolonial que se incorpora para connotar de historicidad a las relaciones observadas (entre el discurso de autonomía, la maternidad subrogada y la tecnología) es un elemento para comprender el funcionamiento del poder capitalista que permea a todas las relaciones por su carácter global. El capitalismo eurocentrado sentó sus bases en la naturalización de la colonialidad del poder. La autonomía de los ciudadanos para elegir sus propias metas y la reproducción de la unidad familiar occidental (que es el patrón de familia burguesa y actual forma de parentesco hegemónica) son fenómenos naturalizados en el discurso y en las instituciones. Optar por una perspectiva decolonial es una apuesta a desestabilizar tal naturalización, desentrañando las lógicas que subyacen en los discursos establecidos. 
Hay una relación clara entre la explotación y la dominación: no toda dominación implica explotación, pero ésta no es posible sin aquella. La dominación es, por lo tanto, sine qua non del poder, de todo poder. Esta es una vieja constante histórica (Quijano, 2007: 323).

En este artículo se analiza la maternidad subrogada colocando el foco de atención en el poder históricamente construido. La dominación de algunos cuerpos sobre otros puede verse en la práctica de la maternidad subrogada. A quiénes corresponden los úteros gestantes y a quiénes corresponden los óvulos y espermas, da cuenta de las relaciones sociales de dominación colonial. La identidad de esos cuerpos está asociada a jerarquías y roles. La identidad racial/étnica de los cuerpos gestantes y de quienes solicitan la gestación tiene su origen en una clasificación social de la población realizada por el poder colonial fundido con el orden capitalista.

El poder es una malla de relaciones sociales de explotación/dominación/conflicto articuladas en el control de diversos ámbitos de la existencia. Los cuatro ámbitos principales son el trabajo (y sus productos); la naturaleza (y sus recursos); el sexo (y su reproducción) y la autoridad (y sus instrumentos) (Quijano, 2007). La maternidad subrogada es una práctica privilegiada para el análisis, en tanto engloba estos cuatro ámbitos de la existencia en donde se articula el poder para el control de dichos ámbitos: 1. La maternidad subrogada es trabajo lucrativo para algunas empresas; 2. es la manipulación (por tecnología) de la naturaleza humana, diseccionada, lo que permite la práctica (separar, unir, trasladar células, etcétera); 3. la reproducción humana es el objeto de la maternidad subrogada; 4. el discurso de autonomía de los sujetos es lo que da autoridad para continuar con el ejercicio de la práctica, es decir, son los sujetos los que eligen libremente participar de la maternidad subrogada y esto la reviste de legitimidad. Pensar la economía de las propiedades vitales desde esta perspectiva decolonial del poder es un aporte para historizar las concepciones/relaciones de los cuerpos y ver cómo operan ciertos discursos liberales.

Este ensayo busca entonces analizar la maternidad subrogada en su contexto históricoeconómico-social, sin desconocer la mercantilización de los cuerpos, la sociedad de clases, la desigualdad racial/étnica y el uso no-neutral de las tecnologías en la nueva geopolítica. El trabajo se compone de tres apartados. El primero describe el funcionamiento de la empresa de medicina reproductiva Procrearte; el segundo puntualiza en el funcionamiento de la esperanza y el biovalor para la economía mundial, es decir el modo en que la esperanza de procrear bebés sanos motoriza la maternidad subrogada y esta práctica va creando circuitos de valor para los fragmentos móviles del cuerpo. El tercer apartado se centra en las nociones de riesgo 
y en el discurso de autonomía mostrando cómo el riesgo impulsa a la maternidad subrogada y el discurso de autonomía la legitima. Por último se plantean interrogantes para agudizar la mirada crítica frente al avance de la tecnomedicina en las economías liberales.

\section{La salud privada como espacio de mercantilización de las relaciones}

Procrearte es una institución que funciona en Argentina, que fue creada en 1999 y cuya sede central se ubica en la Ciudad Autónoma de Buenos Aires. Cuenta con un laboratorio de reproducción asistida que según la misma empresa es el "más sofisticado y mejor desarrollado del país, que le posibilita llevar adelante todas las técnicas de reproducción y ser pioneros en algunas de ellas, acercándole a sus pacientes la mejor tecnología en el mercado nacional, con nivel internacional" (Procrearte, 2018: s/p).

Las tecnologías reproductivas comienzan en los años ochenta en la Argentina. Ya en 1978 se produce en Reino Unido la primera fertilización in vitro exitosa en el mundo, dirigida por Patrick Christopher Steptoe y Robert Geoffrey Edwards. Ocho años después se replica la fecundación in vitro por argentinos. "A partir de este nacimiento, y con el rápido perfeccionamiento y globalización de las técnicas, los servicios de fertilización asistida inician la expansión de su oferta en el país" (Ariza, 2010: 3).

En Procrearte se realizan fertilizaciones in vitro, pero también procedimientos de inseminación artificial, inyección espermática intracitoplasmática; preservan semen, ovocitos y embriones; gestionan ovodonación y espermadonación; y realizan diagnóstico genético preimplantacional. En este caso, interesa analizar particularmente el programa de maternidad subrogada que funciona en dicha institución.

El programa de maternidad subrogada es un servicio hecho en convenio entre la Argentina y México. En el estado de Tabasco desde 1997 se pueden celebrar contratos que regulan la maternidad subrogada. De esta forma, en el estado mexicano opera el "equipo de reclutamiento" que se encarga de encontrar a las "madres subrogadas" y de hacer las indicaciones legales y médicas para garantizar la gestación y la entrega del acta de nacimiento a favor de la madre/padre que ha proporcionado su óvulo/esperma.

Esta empresa cuenta con un Comité de Ética propio el cual aprueba las prácticas en parejas heterosexuales cuyas mujeres no tienen útero, no tienen capacidad reproductiva por causa endometrial, o mujeres con una condición clínica por la cual un embarazo pondría en riesgo su vida, su salud y/o la del bebé (cardiopatías congénitas, diabetes severas, etcétera) o afectaría 
su expectativa de vida. Sólo en esos tres casos se habilita sin restricciones la posibilidad de concretar una maternidad subrogada con esta institución. Asimismo, cuando la pareja es homosexual o no se trata de una pareja sino de una persona sola quien solicita la maternidad subrogada, el Comité de Ética realiza una evaluación particular del caso para habilitar o no dicha práctica. En síntesis, Procrearte es una institución del área de la salud que cuenta con tecnologías reproductivas; como cualquier institución, es una red de relaciones, pero esta tiene la particularidad de gestionar la maternidad subrogada en el sector privado, lo que trae aparejado fenómenos de importancia que se pueden leer en el marco de la actual economía de las propiedades vitales y en clave decolonial.

\section{Bioeconomía y esperanza}

Como ha dicho Michel Foucault (2008), los mecanismos de poder se dirigen al sexo, al cuerpo, a lo que la hace proliferar. $Y$ en este siglo, las tecnologías médicas contemporáneas se ocupan del control de los procesos vitales. Tal como señala Nikolas Rose al comienzo de su libro Políticas de la vida: biomedicina, poder y subjetividad en el siglo XXI

\footnotetext{
la política vital de nuestro siglo no se encuentra delimitada por los polos de la salud y la enfermedad, ni se centra en eliminar patologías para proteger el destino de la nación. Antes bien, se ocupa de nuestra capacidad, cada día mayor, de controlar, administrar, modificar, redefinir y modular las propias capacidades vitales de los seres humanos en cuanto criaturas vivas (2012: 25).
}

Para el autor, en la actualidad la biotecnología redefine al ser biológico y se escapa más allá de lo patológico. Y estas tecnologías de "optimización" se ocupan, entre muchas cosas, de la maternidad/paternidad (Rose, 2012).

No poder gestar un bebé, en esta coyuntura, se construye como un problema plausible de intervención. El proceso vital es una de las razones de ser de las tecnologías médicas. Las antiguas líneas divisorias entre tratamiento, corrección y mejoramiento ya no pueden sostenerse, y algo que no es una enfermedad comienza a ser tratado por la medicina.

La medicina se transforma en tecnomedicina al tener una fuerte dependencia de equipos diagnósticos y terapéuticos de alta complejidad. En el caso de Procrearte, su laboratorio de reproducción asistida, con su "sofisticación" y "desarrollo", funciona como anuncio diseñado para conectar con el público al que va dirigido. El hecho de que sirva como spot publicitario "la 
mejor tecnología en el mercado nacional, con nivel internacional" nos habla de la primacía de la tecnomedicina: son los equipos tecnológicos los que otorgan el reconocimiento y calidad al laboratorio de reproducción asistida.

La concepción y la gestación pasan a ser el interés de empresas como Procrearte, donde el conocimiento es creado a partir de las técnicas, el laboratorio y con especialistas (que son los "pioneros" en las técnicas de reproducción). En este contexto, la existencia vital es foco de control, objeto de formas nuevas de autoridad y conocimiento especializado.

La política contemporánea de la vida en las democracias liberales está marcada por un complejo de mercadización, autonomización y responsabilización. Existe una explotación bioeconómica de los sujetos considerados autónomos y responsables de su propia existencia (aunque justamente esa existencia sea el foco de control de las tecnologías médicas contemporáneas). Esta explotación tiene como condición de posibilidad la fragmentación movible del cuerpo. Los óvulos, espermas, diversos tejidos y fragmentos de ADN pueden hacerse visibles y trasladarse; pueden aislarse, descomponerse, estabilizarse y almacenarse. Estos fragmentos quedan incluso transformados en mercancía.

La maternidad subrogada sería impensable si no pudieran extraerse los óvulos y los espermas, ser trasladados, evaluados (estudios del ADN), transferidos y preservados los embriones obtenidos por fertilización in vitro. Esta fragmentación móvil del cuerpo ha franqueado la entrada a nuevos circuitos: orgánicos, interpersonales, geográficos y financieros: circuitos de vitalidad. $Y$ en estos circuitos de vitalidad es que esos fragmentos van tomando biovalor. El biovalor es el valor que puede extraerse de las propiedades vitales de los procesos vivos (Waldby, 2000). En el caso de la maternidad subrogada, los dos fragmentos del cuerpo que toman importancia bioeconómica son: el útero y el embrión "sin riesgo".

Como se mencionó, el capitalismo colonial/moderno y eurocentrado es un patrón de poder mundial que condiciona los biovalores. El comercio de la maternidad trasciende las fronteras nacionales y se ubica en circuitos transnacionales. Los insumos importados para los laboratorios, el origen de nacionalidad variada de los materiales (manuales, papers, etcétera) para la formación de los especialistas y los convenios binacionales en los programas de maternidad subrogada da cuenta de ello.

En los circuitos se trasluce la desigualdad. Hay división del biovalor: los úteros de las mujeres que llevan la marca de la dominación colonial son mercancía más barata y los embriones blancos son mercancía más costosa. El estado mexicano de Tabasco es un estado con mayoría de población indígena (maya -chol, tzeltal, chontal-) y mestiza. Aunque es el segundo productor nacional de petróleo crudo y es de los principales productores de plátano y cacao, su 
economía es muy frágil comparada con los países más industrializados y con poder financiero. No es casualidad que el "equipo de reclutamiento" (así etiquetado por Procrearte) esté allí ubicado y los úteros que se usen sean de mujeres mexicanas herederas del sufrimiento ocasionado por las relaciones históricamente coloniales. Las "madres subrogadas" son mujeres que reciben un dinero por prestar su cuerpo para gestar un bebé que luego será criado por otra madre/padre. Existe una clara asimetría de las condiciones materiales de existencia entre quien da el dinero para la práctica (padre/madre solicitante de un "vientre") y quien recibe ese dinero (dinero de un monto muy inferior, ya que las empresas como Procrearte, al ser mediadoras, se llevan el mayor porcentaje). Quien da el dinero y el material genético son personas en general hijas de la captura colonial, es decir, blancas o blanqueadas, letradas y propietarias (Segato, 2005). Y quienes reciben el material genético (óvulo fecundado) son mujeres de países empobrecidos para quienes el dinero obtenido en esos nueve meses de embarazo representa mucho más que nueve salarios mínimos que podrían ganar en una actividad productiva.

En India, por ejemplo, todo el proceso de engendrar un bebé puede contratarse por un costo

que oscila entre 3 mil y 10 mil euros. Estos valores suelen quintuplicarse tratándose de los Estados Unidos, Bélgica, Ucrania y Rusia, que observan una formación de precio de mercado debido a las promesas de mayores garantías de salubridad y a las condiciones genético-raciales de las gestantes $y$, aunque este último aspecto suele omitirse, una visita a determinadas páginas de internet permitirá observar la desembozada propaganda eugénica que hacen determinados avisos (Barrancos, 2015: 169).

En estos circuitos globales de intercambio comercial es que se va revistiendo de valor económico (biovalor) a los fragmentos del cuerpo; pero solo algunos cuerpos, por el poder de la colonialidad, son más valorados y deseados que otros. Es la práctica de la biología molecularizada la que permite analizar y descomponer los procesos vitales en sus elementos constitutivos. Pero que pueda aislarlos y "desincrustarlos" de sus sistemas orgánicos de origen no los exime de historicidad. Es la vida misma la que se vuelve pasible de mercantilización, pero se vuelve pasible con su herencia histórica, que marca el camino de esos circuitos de vitalidad desiguales.

En esta explotación bioeconómica que se hace de los cuerpos, la economía de la esperanza toma un papel protagónico. El lenguaje de la esperanza señala la voluntad de superar obstáculos (Brown, 2003; Brown et al., 2000; Franklin, 1997; Good et al., 1990) y genera, incluso, movimiento y organización entre actores (Hess, 2004; Landzelius, 2006). El motor de 
llevar a cabo la maternidad subrogada radica en el deseo de tener un/a hijo/a. El progenitor dará su material genético y nacerá un ser viviente con ese material.

¿Para quién es la maternidad subrogada en Procrearte? "Para aquellos pacientes argentinos que deban realizar esta práctica, con el objetivo de poder aspirar al logro de su hijo", dice textual su página institucional. Y como lo expresa Rose (2012), en el nuevo campo de la biopolítica, donde las intervenciones se ajustan al nivel molecular, la biología no es destino sino oportunidad: descubrir la base biológica de una enfermedad, de la esterilidad, no es resignarse al destino sino abrirse a la esperanza. Tener algunas de las tres "etiologías válidas" para Procrearte (1) es una oportunidad para ingresar al Programa de Maternidad Subrogada. La economía de la esperanza comienza a funcionar cuando se diagnostica un problema y se transforma en posibilidad.

Existe un libro llamado Auxilio, el bebé no llega! (de las autoras Silvia Jadur y Viviana Wainstein) que es promocionado en la página de Procrearte. A continuación, se transcribe un fragmento de la publicidad del libro:

\footnotetext{
Parece sencillo: sólo deben encontrarse un óvulo y un espermatozoide, unirse y empezar a desarrollarse, y después de nueve meses tendremos un bebé en casa. Pero a veces la cosa se complica, y los bebés tardan... o no llegan. $Y$ entonces la cigüeña tiene que pasar antes por el laboratorio.

Desde hace algunas décadas, la ciencia puede ir al rescate de los deseos de tener un hijo y, mediante técnicas de reproducción asistida, colaborar en el deslumbrante proceso de la gestación humana. Fecundación in vitro, óvulos donados, embriones congelados, inyección de espermatozoides; prácticas que parecían de ciencia ficción, hoy son de rutina en los centros de salud. En este libro, las autoras recorren los temas esenciales en torno a la fascinante biografía de un humanito y nos regalan toda su experiencia en aliviar el camino de los buscadores de bebés (subrayado propio).
}

Es el deseo de tener un hijo lo que impulsa a las personas a practicar una maternidad subrogada. Lo que parecía de ciencia ficción es posible. Surge un nuevo fenómeno en la tecnomedicina: la esperanza de concretar el deseo con la tecnología. La creencia de que la ciencia cumple el deseo, es otra de las condiciones de posibilidad de la bioeconomía.

\section{Riesgo y autonomía}


"El lenguaje del riesgo recodifica el tratamiento de los problemas. Nuevas zonas de intervención se hacen visibles y la 'administración del riesgo' se añade a las exigencias de individuos y autoridades" (Bianchi, 2015: 87). En la maternidad subrogada existen dos grandes riesgos: el del embrión y el de la mujer que solicita un vientre para gestar. Que la salud de la mujer corra riesgo en la gestación es uno de los requisitos para ingresar al Programa de Procrearte. Cuando el embarazo pone en riesgo la vida o la salud de la mujer, entonces el comité de ética aprueba su solicitud. El otro riesgo es el del embrión. La empresa realiza una suerte de "selección de calidad" de los óvulos, espermatozoides y de embriones. Seleccionan los embriones sin riesgo de tener enfermedades o malformaciones. Para Rose (2012), "el lenguaje del riesgo genético nos empieza a proporcionar gradualmente una grilla de percepción que guía las decisiones respecto de cómo conducir la propia vida, tener hijos, contraer matrimonio o transitar una carrera" (p. 256). La noción de riesgo genético forma parte de las agendas de estas instituciones que incorporan como clientes a mujeres con "riesgo" en el embarazo y les garantizan una maternidad subrogada "sin riesgo".

En relación a la ciudadanía biológica, propia de las sociedades contemporáneas, Rose (2012) nos dice que esta

depende de que la ciencia del presente produzca curas o tratamientos en el futuro cercano, y también lo espera. Esperar, en este sentido, no se reduce a desear y anticipar: postula cierto futuro posible y deseable, que requiere actuar en el presente para su realización ( $p$. 297).

Las mujeres y los hombres clientes de Procrearte deben actuar en este presente para concretar la llegada futura del/a hijo/a. Parafraseando la publicidad del libro mencionado, el accionar presente hace que llegue el "humanito" y así se alivia "el camino de los buscadores de bebés".

En esta sociedad donde los "buscadores de bebés" hacen uso de la tecnomedicina en una bioeconomía global, se construye ciudadanía.

Construir ciudadanos entraña redefinir el modo en que las autoridades (sean autoridades políticas, personal médico, profesionales legales y penales, potenciales empleadores o compañías aseguradoras) entienden a las personas en función de categorías... estas categorías organizan la mirada diagnóstica, forense e interpretativa de diferentes grupos de profesionales y especialistas. La clasificación de este tipo divide y unifica a la vez; delimita las fronteras de quién recibe qué tratamiento: castigo, terapia, empleo, seguridad, 
beneficios, recompensas. Y los unifica dentro de una categoría, pasando por alto sus diferencias específicas (p. 284).

Para Procrearte, quienes pueden recibir tratamiento son las parejas heterosexuales con las tres etiologías ya enumeradas. Y las "madres subrogadas" son las mujeres del estado mexicano de Tabasco. En esta política de la empresa los agentes institucionales (empresarios y especialistas) delimitan quién recibe qué. Difiriendo un poco con la cita de Rose, quien afirma que en la construcción de ciudadanía se pasan por alto diferencias, para el caso de la maternidad subrogada no se pasa por alto la diferencia de clase y étnica-racial, ya que es esta la que unifica al grupo que ingresa al Programa.

Subyacente a la idea política de ciudadanía se encuentra el discurso de la autonomía, heredera de la visión tributaria del liberalismo individualista y la ciudadanía ilustrada. La autonomía que realza el libre albedrío autodirigido es la que da legitimidad a la maternidad subrogada, porque quien dispone de su propio cuerpo es la mujer y es ella la que elige que la fecunden o es ella la que elige pagar para que otra mujer se fecunde.

Existiendo en esta empresa de medicina reproductiva una serie de consentimientos informados a firmar por ambas partes, hay una acción de las personas en nombre de sus propios intereses. Las personas tienen la autoridad y autonomía de agenciar en la maternidad subrogada.

\section{Reflexiones finales}

En la geopolítica contemporánea, las propiedades vitales se convierten en mercancía y surgen empresas como Procrearte que lucran e ingresan en los circuitos de vitalidad a partir de la maternidad subrogada. Observando la división del trabajo mundial de esta práctica, podemos observar que existen criterios de origen clasista y racista al momento de elegir a las "madres subrogadas" que gestan a bebés que serán inscriptos en actas de nacimientos para otras personas. $Y$ la posibilidad de pagar por una maternidad subrogada en "Procrearte" queda circunscripta y reducida a las clases medias-altas y altas. Entonces, surge la pregunta: ¿En qué medida los cuerpos de las mujeres más vulnerables son usados para beneficio empresarial? El discurso de la autonomía de esas mujeres es lo que aplaca las posibles críticas. Sus firmas en los consentimientos informados son un aval a gestar embriones por dinero. 
En esta sociedad con grandes transformaciones en la tecnomedicina se puede concluir, desde una lectura feminista, que las mujeres son libres de decidir sobre su propio cuerpo y nadie podrá prohibir que las mujeres se fecunden por medio de estas tecnologías; pero también puede concluirse que las mismas mujeres están siendo usadas como objetos experimentales por las empresas tecnomédicas lucrativas. Ambas posturas tienen sus argumentos. Sea como fuere, nuevos interrogantes se abren en el derrotero del control de los procesos vitales en las bioeconomías liberales.

\section{Notas}

(1) No tener útero, no tener capacidad reproductiva por causa endometrial o tener una condición clínica por la cual el embarazo pondría en riesgo la propia vida, la salud y/o la del bebé (cardiopatías congénitas, diabetes severa, etcétera) o afecten su expectativa de vida.

\section{Bibliografía}

Ariza, L. (2010). La procreación como evento natural o tecnológico: repertorios decisorios acerca del recurso a la reproducción asistida en parejas infértiles de Buenos Aires. Eä Journal. 2(1).

Barrancos, D. (2015). Dilemas éticos de la reproducción tecno-mediada: una reflexión más allá de la cosmovisión religiosa. Sociedad y religión, 25(44), 155-179. Recuperado de https://bit.ly/2tJmFFU.

Bianchi, E. (2012). El problema del riesgo. Notas para una reflexión sobre los aportes de la usina genealógico en torno al concepto de riesgo en salud mental. Espacios. Estudios de Biopolítico, 7, pp. 84-97.

Brown, N. (2003). Hope against hype-accountability in biopasts, presents and futures. Science Studies, 16(2), 3-21.

Brown, N.; Rappert, B. \& Webster, A. (2000). Introducing contested futures: From looking into the future to looking at the future. En Webster, A.; Brown, N. \& Rappert, B. (eds). Contested futures: A sociology of prospective techno-science. USA: Routledge.

Foucault, M. (2008). Historia de la sexualidad 1: la voluntad del saber (Segunda edición). Buenos Aires: Siglo XXI Editores. 
Franklin, S. (1997). Embodied progress: A cultural account of assisted conception. London: Routledge.

Good, M-J. D.; Good, B.; Schaefer, C. \& Lind, S. E. (1990). American oncology and the discourse on hope. Culture, Medicine and Psychiatry, 14(1), 59-79.

Hess, D.J. (2004). Health, the environment and social movements. Science as Culture, 13(4), $421-7$.

Landzelius, K. (2006). Introduction: Patient organization movements and new metamorphoses in patienthood. Social Science \& Medicine, 62(3), 529-537.

Procrearte (2018). Programa de Maternidad Subrogada. Recuperado de http://www.procrearte.com.

Quijano, A. (2007). Colonialidad del poder y clasificación social. En Castro-Gómez, S. y Grosfoguel, R. (Comps.). El giro decolonial: reflexiones para una diversidad epistémica más allá del capitalismo global (pp. 93-126). Bogotá: Siglo del Hombre Editores; Universidad Central, Instituto de Estudios Sociales Contemporáneos y Pontificia Universidad Javeriana, Instituto Pensar.

Rose, N. (2012). Políticas de la vida: biomedicina, poder y subjetividad en el siglo XXI. La Plata: UNIPE.

Segato, R. (2015). La crítica de la colonialidad en ocho ensayos. Buenos Aires: Prometeo Libros

Waldby, C. (2000). The Visible Human Project: Informatic bodies and posthuman medicine. London, New York: Routledge. 